九州大学学術情報リポジトリ

Kyushu University Institutional Repository

\title{
A Single-Step Characteristic-Curve Finite Element Scheme of Second Order in Time for the Incompressible Navier-Stokes Equations
}

Notsu, Hirofumi

Faculty of Mathematics, Kyushu University

Tabata, Masahisa

Faculty of Mathematics, Kyushu University

http://hdl. handle. net/2324/9351

出版情報：Journal of Scientific Computing. 38 (1)，pp.1-14，2008-07-11. Springer バージョン：

権利関係 : 


\section{MHF Preprint Series}

Kyushu University

21st Century COE Program

Development of Dynamic Mathematics with

High Functionality

\section{A characteristic-curve finite element scheme of single step and second order in time increment for the Navier-Stokes equations}

\section{H. Notsu \& M. Tabata}

MHF 2008-4

( Received February 21, 2008 )

Faculty of Mathematics

Kyushu University

Fukuoka, JAPAN 


\title{
A Characteristic-Curve Finite Element Scheme of Single Step and Second Order in Time Increment for the Navier-Stokes Equations
}

\author{
Hirofumi NOTSU* and Masahisa TABATA ${ }^{\dagger}$ \\ Faculty of Mathematics, Kyushu University, Fukuoka 812-8581, Japan
}

\begin{abstract}
In this paper we present a new characteristic-curve finite element scheme of single step and second order in time increment for the nonstationary Navier-Stokes equations. After supplying correction terms in the variational formulation, we prove that the scheme is of second order in time increment. The convergence rate of the scheme is numerically recognized by computational results.
\end{abstract}

\section{Introduction}

In this paper we present a characteristic-curve finite element scheme of single step and second order in time increment for the Navier-Stokes equations. In devising numerical schemes for the Navier-Stokes equations, a key issue is how to approximate the nonlinear convection term. Especially it is crucial in the computation of high-Reynolds number problems, where the standard Galerkin approximation leads to disastrous oscillating results. Many kinds of approximations have been developed based on ideas such as upwinding, streamline diffusion, least square, characteristic curve, and so on. (See [1] , [3], [4] , [5] , [7] , [8] , [11] , [12] , [13], [14], [15], [16], [19], [20], [22] , [25] and references there in).

We focus on the approximation based on characteristic-curve method. The idea of the characteristiccurve method is to consider the trajectory of the fluid particle and discretize the material derivative term along the trajectory. The procedure is natural from the physical point of view. Furthermore, the matrix for the system of linear equations is symmetric, which leads to easy solvers for the system. Characteristiccurve finite element schemes for the Navier-Stokes equations of first order in time increment have been developed and analysed, see [14], [15], [19]. A scheme of second order in time increment has been presented and analysed by Boukir et al. [5]. They use a two-step method and approximate the material derivative by the values of two previous steps along the trajectory.

Here we propose a scheme of second order in time increment and of single step. The material derivative is approximated in the Crank-Nicolson way along the trajectory. The original idea of the approximation was developed in [17] for the convection-diffusion equations, which is extended to the Naveir-Stokes equations in this paper. As is pointed out in [17], in the Crank-Nicolson approximation on the trajectory, an additional correction term is indispensable to realize a second order accuracy. After supplying correction terms for the Navier-Stokes equations, the scheme is proved to be of second order in time increment. In the case of the Navier-Stokes equations the velocity is unknown and the obtained scheme becomes nonlinear. For the solution we present an internal iteration procedure which consists of solving a series of Stokes type equations. The scheme has such advantages that it is of second order in time increment and that every matrix to be treated is symmetric.

We use the function spaces $L^{2}(\Omega)$ and $H^{1}(\Omega)$, and their subspaces $H_{0}^{1}(\Omega)$ and

$$
L_{0}^{2}(\Omega) \equiv\left\{\phi \in L^{2}(\Omega) ; \int_{\Omega} \phi d x=0\right\} .
$$

*notsu@math.kyushu-u.ac.jp

†tabata@math.kyushu-u.ac.jp 
We denote by $(\cdot, \cdot)$ the $L^{2}(\Omega)$-inner products in the scalar- and vector-valued function spaces. For any normed space $X$ the norm is denoted by $\|\cdot\|_{X}$. When $X=L^{2}(\Omega)^{d}$ or $L^{2}(\Omega)$, we omit the subscript and denote it simply by $\|\cdot\|$. The dual pairing between $X$ and the dual space $X^{\prime}$ is denoted by $\langle\cdot, \cdot\rangle$. The partial derivative $\partial u / \partial x_{i}$ of a function $u$ is simply denoted by $u_{, i}$ and the Einstein convention $a_{i} b_{i}$ is used in place of $\sum_{i=1}^{d} a_{i} b_{i}$.

The outline of the paper is as follows. We present a characteristic-curve finite element scheme for the Navier-Stokes equations in Section 2. In Section 3 the consistency of the scheme is proved to be of second order in time increment. In the last section we show some numerical examples to observe the convergence rate.

\section{A Characteristic-Curve Finite Element Scheme}

In this section we present a characteristic-curve finite element scheme for the Navier-Stokes equations. It is of single step and second order in time increment.

Let $\Omega \subset \mathbb{R}^{d}(d=2,3)$ be a bounded domain and $T$ be a positive constant. We consider the nonstationary Navier-Stokes problem subject to the homogeneous Dirichlet boundary condition; find $(u, p)$ : $\Omega \times(0, T) \rightarrow \mathbb{R}^{d} \times \mathbb{R}$ such that

$$
\left\{\begin{array}{rlrl}
\frac{\partial u}{\partial t}+(u \cdot \nabla) u-\nabla(2 v D(u))+\nabla p & =f & & \text { in } \Omega \times(0, T), \\
\nabla \cdot u=0 & & \text { in } \Omega \times(0, T), \\
u=0 & & \text { on } \Gamma \times(0, T), \\
u=u^{0} & & \text { in } \Omega, \text { at } t=0,
\end{array}\right.
$$

where $u$ is the velocity, $p$ is the pressure, $f$ is an exterior force, $u^{0}$ is an initial velocity, $v(>0)$ is a viscosity, $\Gamma \equiv \partial \Omega$ is the boundary of $\Omega, D(u)$ is the strain-rate tensor defined by

$$
D_{i j}(u) \equiv \frac{1}{2}\left(u_{i, j}+u_{j, i}\right)
$$

and

$$
[\nabla(2 v D(u))]_{i} \equiv 2 v D_{i j, j}(u) .
$$

In order to present our scheme for (2.1) we prepare the following. For a time increment $\Delta t$ and velocities $u$ and $w: \Omega \rightarrow \mathbb{R}^{d}$, we define $X_{1}(u, \Delta t)$ and $X_{2}(u, w, \Delta t)$ by

$$
\begin{aligned}
X_{1}(u, \Delta t)(x) & \equiv x-u(x) \Delta t, \\
X_{2}(u, w, \Delta t)(x) & \equiv x-\{u(x)+w(x-w(x) \Delta t)\} \frac{\Delta t}{2},
\end{aligned}
$$

respectively. We use the symbol $\circ$ to designate the composition of functions, e.g., for a function $\psi$ defined in $\Omega$

$$
\left(\psi \circ X_{1}(u, \Delta t)\right)(x) \equiv \psi(x-u(x) \Delta t) .
$$

For $n \in \mathbb{N} \cup\{0\}$ we set

$$
t^{n} \equiv \begin{cases}\Delta t_{0}+(n-1) \Delta t & (n \geq 1), \\ 0 & (n=0),\end{cases}
$$

where $\Delta t_{0}$ is another time increment used only in the first step of the computation. Let $N_{T} \equiv[(T-$ $\left.\left.\Delta t_{0}\right) / \Delta t\right]+1$ be a total step number, $\mathscr{T}_{h} \equiv\{K\}$ be a triangulation of $\Omega$. We define $\Omega_{h}$ by

$$
\Omega_{h} \equiv \text { int } \bigcup\left\{K ; K \in \mathscr{T}_{h}\right\}
$$


and $\Gamma_{h} \equiv \partial \Omega_{h}$. We set finite element spaces,

$$
\begin{aligned}
X_{h} & \equiv\left\{v_{h} \in C^{0}\left(\bar{\Omega}_{h}\right)^{d} ;\left.v_{h}\right|_{K} \in P_{2}(K), \forall K\right\}, & & V_{h} \equiv X_{h} \cap H_{0}^{1}\left(\Omega_{h}\right), \\
M_{h} & \equiv\left\{q_{h} \in C^{0}\left(\bar{\Omega}_{h}\right) ;\left.q_{h}\right|_{K} \in P_{1}(K), \forall K\right\}, & & Q_{h} \equiv M_{h} \cap L_{0}^{2}\left(\Omega_{h}\right),
\end{aligned}
$$

and define interpolation operators,

$$
\Pi_{h}^{(1)}: C^{0}\left(\bar{\Omega}_{h}\right) \rightarrow M_{h}, \quad \Pi_{h}^{(2)}: C^{0}\left(\bar{\Omega}_{h}\right)^{d} \rightarrow X_{h} .
$$

For $u, w$ and $\zeta \in H_{0}^{1}\left(\Omega_{h}\right)^{d}, p$ and $q \in H^{1}\left(\Omega_{h}\right)$ and $r, f$ and $g \in L^{2}\left(\Omega_{h}\right)$ we define linear forms $\mathscr{A}_{h 1}(u, w, r)$, $\mathscr{A}_{h 2}(u, \zeta, w, p, q), \mathscr{F}_{h 1} f$ and $\mathscr{F}_{h 2}(f, g, w)$ on $V_{h}$ and $\mathscr{B}_{h} u$ on $Q_{h}$ by

$$
\begin{aligned}
\mathscr{A}_{h 1}(u, w, r) & \equiv \mathscr{M}_{h 1}\left(u, w ; \Delta t_{0}\right)+\mathscr{D}_{h 1} u+\mathscr{C}_{h 1} r, \\
\mathscr{A}_{h 2}(u, \zeta, w, p, q) & \equiv \mathscr{M}_{h 2}(u, \zeta, w ; \Delta t)+\mathscr{D}_{h 2}(u, w)+\mathscr{C}_{h 2}(w, p, q), \\
\left\langle\mathscr{B}_{h} u, q_{h}\right\rangle & \equiv-\left(\nabla \cdot u, q_{h}\right), \quad\left\langle\mathscr{F}_{h 1} f, v_{h}\right\rangle \equiv\left(f, v_{h}\right), \\
\left\langle\mathscr{F}_{h 2}(f, g, w), v_{h}\right\rangle & \equiv \frac{1}{2}\left(f+g \circ X_{1}(w, \Delta t), v_{h}\right),
\end{aligned}
$$

where

$$
\begin{aligned}
\left\langle\mathscr{M}_{h 1}\left(u, w ; \Delta t_{0}\right), v_{h}\right\rangle & \equiv\left(\frac{u-w \circ X_{1}\left(w, \Delta t_{0}\right)}{\Delta t_{0}}, v_{h}\right), \\
\left\langle\mathscr{M}_{h 2}(u, \zeta, w ; \Delta t), v_{h}\right\rangle & \equiv\left(\frac{u-w \circ X_{2}(\zeta, w, \Delta t)}{\Delta t}, v_{h}\right), \\
\left\langle\mathscr{D}_{h 1} u, v_{h}\right\rangle & \equiv 2 v\left(D(u), D\left(v_{h}\right)\right), \quad\left\langle\mathscr{C}_{h 1} r, v_{h}\right\rangle \equiv-\left(\nabla \cdot v_{h}, r\right), \\
\left\langle\mathscr{D}_{h 2}(u, w), v_{h}\right\rangle & \equiv v\left(D(u)+D(w) \circ X_{1}(w, \Delta t), D\left(v_{h}\right)\right)+v \Delta t\left(D_{i j}(w) w_{k, j}, v_{h i, k}\right), \\
\left\langle\mathscr{C}_{h 2}(w, p, q), v_{h}\right\rangle & \equiv \frac{1}{2}\left(\nabla p+\nabla q \circ X_{1}(w, \Delta t), v_{h}\right) .
\end{aligned}
$$

For $\left\{u^{n}\right\}_{n=0}^{N_{T}} \subset H_{0}^{1}\left(\Omega_{h}\right)^{d},\left\{p^{n}\right\}_{n=1}^{N_{T}} \subset H^{1}\left(\Omega_{h}\right)$ and $\left\{f^{n}\right\}_{n=1}^{N_{T}} \subset L^{2}\left(\Omega_{h}\right)^{d}$, linear forms $\mathscr{A}_{h}^{n}(u, p)$ and $\mathscr{F}_{h}^{n}(f, u)$ on $V_{h}$ are defined by

$$
\begin{aligned}
\mathscr{A}_{h}^{n}(u, p) & \equiv \begin{cases}\mathscr{A}_{h 2}\left(u^{n}, u^{n}, u^{n-1}, p^{n}, p^{n-1}\right) & (n \geq 2), \\
\mathscr{A}_{h 1}\left(u^{1}, u^{0}, p^{1}\right) & (n=1),\end{cases} \\
\mathscr{F}_{h}^{n}(f, u) & \equiv \begin{array}{ll}
\mathscr{F}_{h 2}\left(f^{n}, f^{n-1}, u^{n-1}\right) & (n \geq 2), \\
\mathscr{F}_{h 1} f^{1} & (n=1) .
\end{array}
\end{aligned}
$$

In order to unify the notation we put $\mathscr{B}_{h}^{n} u \equiv \mathscr{B}_{h} u^{n}$. For a given continuous function $f$ we set $f_{h}^{n} \equiv$ $\Pi_{h}^{(2)} f\left(t^{n}\right)$.

We now present the scheme for (2.1); find $\left\{\left(u_{h}^{n}, p_{h}^{n}\right)\right\}_{n=1}^{N_{T}} \subset V_{h} \times Q_{h}$ such that

$$
\left\{\begin{aligned}
\mathscr{A}_{h}^{n}\left(u_{h}, p_{h}\right) & =\mathscr{F}_{h}^{n}\left(f_{h}, u_{h}\right) & & \text { in } V_{h}^{\prime}, \\
\mathscr{B}_{h}^{n} u_{h} & =0 & & \text { in } Q_{h}^{\prime}, \\
u_{h}^{0} & =\Pi_{h}^{(2)} u^{0} . & &
\end{aligned}\right.
$$


For $n \geq 2$ this is equivalent to the equations,

$$
\left\{\begin{array}{l}
\left(\frac{u_{h}^{n}-u_{h}^{n-1} \circ X_{2}\left(u_{h}^{n}, u_{h}^{n-1}, \Delta t\right)}{\Delta t}, v_{h}\right) \\
\quad+v\left(D\left(u_{h}^{n}\right)+D\left(u_{h}^{n-1}\right) \circ X_{1}\left(u_{h}^{n-1}, \Delta t\right), D\left(v_{h}\right)\right)+v \Delta t\left(D_{i j}\left(u_{h}^{n-1}\right) u_{h k, j}^{n-1}, v_{h i, k}\right) \\
\quad+\frac{1}{2}\left(\nabla p_{h}^{n}+\nabla p_{h}^{n-1} \circ X_{1}\left(u_{h}^{n-1}, \Delta t\right), v_{h}\right) \\
\quad=\frac{1}{2}\left(f_{h}^{n}+f_{h}^{n-1} \circ X_{1}\left(u_{h}^{n-1}, \Delta t\right), v_{h}\right), \quad \forall v_{h} \in V_{h}, \\
\left(\nabla \cdot u_{h}^{n}, q_{h}\right)=0, \quad \forall q_{h} \in Q_{h} .
\end{array}\right.
$$

In the next section the scheme is shown to be of second order in $\Delta t$ for $n \geq 2$, and of first order in $\Delta t_{0}$ for $n=1$. By taking $\Delta t_{0}=O\left(\Delta t^{2}\right)$, the whole scheme becomes of second order in time increment $\Delta t$.

Remark 2.1 (i) For $v_{h} \in V_{h}$ and $q_{h} \in Q_{h}$ it holds that

$$
\left\langle\mathscr{C}_{h 1} q_{h}, v_{h}\right\rangle=\left\langle\mathscr{B}_{h} v_{h}, q_{h}\right\rangle
$$

i.e., $\mathscr{C}_{h 1}=\mathscr{B}_{h}^{\prime}$ on $Q_{h}$, though $\mathscr{C}_{h 1}$ is defined on $L^{2}\left(\Omega_{h}\right)$.

(ii) In $\mathscr{A}_{h}^{n}(n \geq 2)$, we need $u^{n-1}$ and $p^{n-1}$ to get $u^{n}$ and $p^{n}$. If $\mathscr{A}_{h 2}$ were used when $n=1$, we would need $p^{0}$, which is not given as the initial condition in the Navier-Stokes equations. This is the reason why we use $\mathscr{A}_{h 1}$ at $n=1$. In the case of the convection-diffusion equation, such fact does not occur.

Since the scheme is nonlinear in $u_{h}^{n}$ for $n \geq 2$, we prepare an internal iteration procedure. Let $\left\{\left(w_{h}^{k}, r_{h}^{k}\right)\right\}_{k=1}^{\infty} \subset V_{h} \times Q_{h}$ be the solution of

$$
\left\{\begin{array}{rlrl}
\mathscr{A}_{h 2}\left(w_{h}^{k}, w_{h}^{k-1}, u_{h}^{n-1}, r_{h}^{k}, p_{h}^{n-1}\right) & =\mathscr{F}_{h 2}\left(f_{h}^{n}, f_{h}^{n-1}, u_{h}^{n-1}\right) & & \text { in } V_{h}^{\prime}, \\
\mathscr{B}_{h} w_{h}^{k} & =0 & & \text { in } Q_{h}^{\prime}, \\
w_{h}^{0} & =u_{h}^{n-1} . &
\end{array}\right.
$$

$\left(u_{h}^{n}, p_{h}^{n}\right)$ is obtained as the limit of the sequence $\left\{\left(w_{h}^{k}, r_{h}^{k}\right)\right\}_{k=1}^{\infty}$. In the real computation if the convergence criterion,

$$
\frac{\left\|w_{h}^{k}-w_{h}^{k-1}\right\|_{H^{1}\left(\Omega_{h}\right)^{d}}+\left\|r_{h}^{k}-r_{h}^{k-1}\right\|_{L^{2}\left(\Omega_{h}\right)}}{\left\|w_{h}^{k}\right\|_{H^{1}\left(\Omega_{h}\right)^{d}}+\left\|r_{h}^{k}\right\|_{L^{2}\left(\Omega_{h}\right)}}<\varepsilon_{I}
$$

is satisfied for some $k$, we set $\left(u_{h}^{n}, p_{h}^{n}\right) \equiv\left(w_{h}^{k}, r_{h}^{k}\right)$. Here $\varepsilon_{I}$ is a sufficiently small positive constant. We note that (2.4) is a linear problem in $w_{h}^{k}$ and $r_{h}^{k}$ whose matrix is symmetric.

Remark 2.2 One can choose other finite element spaces $V_{h} \times Q_{h}$ satisfying the inf-sup condition [6], [10] and $Q_{h} \subset H^{1}\left(\Omega_{h}\right)$.

Remark 2.3 Scheme (2.3) requires that $Q_{h}$ is a subset of $H^{1}\left(\Omega_{h}\right)$, because the pressure term is written in a strong form. Using a weak form for the pressure, which requires only $Q_{h} \subset L^{2}\left(\Omega_{h}\right)$, we can derive a scheme,

$$
\left\{\begin{aligned}
\tilde{\mathscr{A}}_{h}^{n}\left(u_{h}, p_{h}\right) & =\mathscr{F}_{h}^{n}\left(f_{h}, u_{h}\right) & & \text { in } V_{h}^{\prime}, \\
\mathscr{B}_{h}^{n} u_{h} & =0 & & \text { in } Q_{h}^{\prime}, \\
u_{h}^{0} & =\Pi_{h}^{(2)} u^{0}, & &
\end{aligned}\right.
$$

where

$$
\tilde{\mathscr{A}}_{h}^{n}(u, p) \equiv \begin{cases}\mathscr{M}_{h 2}\left(u^{n}, u^{n}, u^{n-1} ; \Delta t\right)+\mathscr{D}_{h 2}\left(u^{n}, u^{n-1}\right)+\tilde{\mathscr{C}}_{h 2}\left(u^{n-1}, p^{n}, p^{n-1}\right) & (n \geq 2) \\ \mathscr{M}_{h 1}\left(u^{1}, u^{0} ; \Delta t_{0}\right)+\mathscr{D}_{h 1} u^{1}+\mathscr{C}_{h 1} p^{1} & (n=1)\end{cases}
$$




$$
\left\langle\tilde{\mathscr{C}}_{h 2}(w, p, q), v_{h}\right\rangle \equiv-\frac{1}{2}\left(\nabla \cdot v_{h}, p+q \circ X_{1}(w, \Delta t)\right)-\frac{\Delta t}{2}\left(q w_{i, j}, v_{h j, i}\right) .
$$

The last term of (2.7) is a correction term for second order accuracy in $\Delta t$. This scheme is proved to be of second order in $\Delta t$ in a similar way to scheme (2.3) by using the analysis in the next section. Numerical experiments, however, show that scheme (2.6) is not so stable. In fact, we could not get solutions for $v \leq 10^{-2}$ in Example 1 of Section 4 because of oscillation. Hence we do not use this scheme.

\section{Consistency of the Scheme}

In this section we assume $\Omega_{h}=\Omega$ for the sake of simplicity. For an integer $n\left(2 \leq n \leq N_{T}\right)$, we set

$$
t^{n-1 / 2} \equiv \frac{1}{2}\left(t^{n}+t^{n-1}\right) .
$$

For a function $\psi$ on $\Omega \times(0, T)$ and $m \in \mathbb{N} \cup\{\mathbb{N}-1 / 2\} \cup\{0\}\left(m \leq N_{T}\right), \psi^{m}$ means

$$
\psi^{m} \equiv \psi\left(\cdot, t^{m}\right)
$$

Proposition 3.1 (consistency) Suppose that $f$ is a sufficiently smooth function, $(u, p)$ is the sufficiently smooth solution of (2.1) and $X_{1}\left(u^{n-1}, \Delta t\right)(\Omega)$ and $X_{2}\left(u^{n}, u^{n-1}, \Delta t\right)(\Omega) \subset \Omega$. Then for any $v_{h} \in V_{h}$ it holds that

$$
\begin{aligned}
\left\langle\mathscr{A}_{h 2}\left(u^{n}, u^{n}, u^{n-1}, p^{n}, p^{n-1}\right)-\mathscr{F}_{h 2}\left(f^{n}, f^{n-1}, u^{n-1}\right), v_{h}\right\rangle & =O\left(\Delta t^{2}\right)\left\|v_{h}\right\|, \\
\left\langle\mathscr{A}_{h 1}\left(u^{1}, u^{0}, p^{1}\right)-\mathscr{F}_{h 1} f^{1}, v_{h}\right\rangle & =O\left(\Delta t_{0}\right)\left\|v_{h}\right\| .
\end{aligned}
$$

We prepare some lemmas for the proof. The first one is trivial, but it is often used.

Lemma 3.1 For a smooth function $f$ it holds that

$$
\begin{aligned}
\frac{1}{2}(f(t)+f(t-\Delta t)) & =f\left(t-\frac{\Delta t}{2}\right)+O\left(\Delta t^{2}\right), \\
\frac{f(t)-f(t-\Delta t)}{\Delta t} & =f^{\prime}\left(t-\frac{\Delta t}{2}\right)+O\left(\Delta t^{2}\right) .
\end{aligned}
$$

Let $u: \Omega \times(0, T) \rightarrow \mathbb{R}^{d}$ be a smooth function. For a point $x \in \Omega$, we denote by $X(\cdot ; x):(0, T) \rightarrow \mathbb{R}^{d}$ the solution of the ordinary differential equation,

$$
\left\{\begin{array}{l}
X^{\prime}(t)=u(X(t), t) \quad \text { in }\left(t^{n-1}, t^{n}\right), \\
X\left(t^{n}\right)=x
\end{array}\right.
$$

We denote the material derivation by

$$
\frac{D}{D t} \equiv \frac{\partial}{\partial t}+u \cdot \nabla .
$$

We note that the material derivative of a function $f: \Omega \times(0, T) \rightarrow \mathbb{R}$ is written as

$$
\frac{D f}{D t}(X(t), t)=\frac{d}{d t} f(X(t), t) .
$$

Setting

$$
Y_{1}(u, \Delta t)(x) \equiv \frac{x+X_{1}(u, \Delta t)(x)}{2},
$$




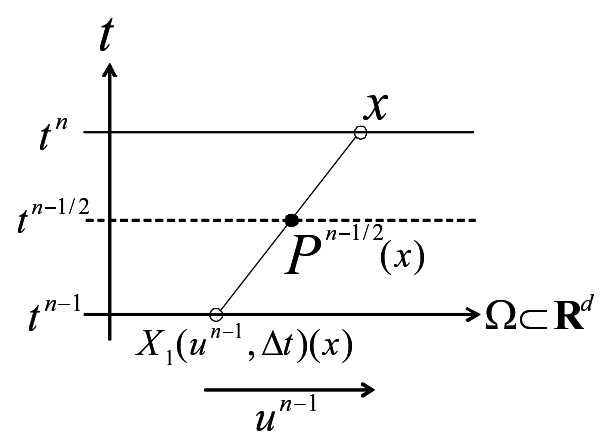

Fig. 1: The evaluation point for the consistency

we evaluate the equations at a point

$$
P^{n-1 / 2}(x) \equiv\left(Y_{1}\left(u^{n-1}, \Delta t\right)(x), t^{n-1 / 2}\right) .
$$

Using the approximation $X_{2}$ for $X\left(t^{n-1}\right)$, we can construct a second order discretization of the material derivative as follows.

Lemma 3.2 Let $u$ be a sufficiently smooth function and $X_{2}\left(u^{n}, u^{n-1}, \Delta t\right)(\Omega) \subset \Omega$. Then it holds that

$$
\frac{u^{n}(x)-u^{n-1} \circ X_{2}\left(u^{n}, u^{n-1}, \Delta t\right)(x)}{\Delta t}=\frac{D u}{D t}\left(P^{n-1 / 2}(x)\right)+O\left(\Delta t^{2}\right) .
$$

Proof Let $X$ be the solution of (3.3). Substituting $u$ into $f$ in (3.4) and using (3.2b), we have

$$
\frac{D u}{D t}\left(X\left(t^{n-1 / 2}\right), t^{n-1 / 2}\right)=\frac{u^{n}\left(X\left(t^{n}\right)\right)-u^{n-1}\left(X\left(t^{n-1}\right)\right)}{\Delta t}+O\left(\Delta t^{2}\right) .
$$

Since the Heun method is of second order in time increment, we have

$$
\begin{aligned}
X\left(t^{n-1} ; x\right) & =x-\left\{u^{n}(x)+u^{n-1}\left(x-u^{n}(x) \Delta t\right)\right\} \frac{\Delta t}{2}+O\left(\Delta t^{3}\right) \\
& =x-\left\{u^{n}(x)+u^{n-1}\left(x-u^{n-1}(x) \Delta t\right)\right\} \frac{\Delta t}{2}+O\left(\Delta t^{3}\right) \\
& =X_{2}\left(u^{n}, u^{n-1}, \Delta t\right)(x)+O\left(\Delta t^{3}\right) .
\end{aligned}
$$

On the other hand, by (3.2a), it holds that

$$
X\left(t^{n-1 / 2} ; x\right)=Y_{1}\left(u^{n-1}, \Delta t\right)(x)+O\left(\Delta t^{2}\right) .
$$

Combining (3.8) and (3.9) with (3.7), we get (3.6).

Lemma 3.3 Suppose that $u, f: \Omega \times(0, T) \rightarrow \mathbb{R}^{d}$ and $p: \Omega \times(0, T) \rightarrow \mathbb{R}$ are sufficiently smooth functions and $X_{1}\left(u^{n-1}, \Delta t\right)(\Omega)$ and $X_{2}\left(u^{n}, u^{n-1}, \Delta t\right)(\Omega) \subset \Omega$. Then for any $x \in \Omega$ it holds that

$$
\begin{aligned}
& \frac{u^{n}-u^{n-1} \circ X_{2}\left(u^{n}, u^{n-1}, \Delta t\right)}{\Delta t}(x)-v\left\{\nabla D\left(u^{n}\right)+\nabla D\left(u^{n-1}\right) \circ X_{1}\left(u^{n-1}, \Delta t\right)\right\}(x) \\
& +\frac{1}{2}\left\{\nabla p^{n}+\nabla p^{n-1} \circ X_{1}\left(u^{n-1}, \Delta t\right)\right\}(x)-\frac{1}{2}\left\{f^{n}+f^{n-1} \circ X_{1}\left(u^{n-1}, \Delta t\right)\right\}(x) \\
= & \left(\frac{D u}{D t}-2 v \nabla D(u)+\nabla p-f\right)\left(P^{n-1 / 2}(x)\right)+O\left(\Delta t^{2}\right),
\end{aligned}
$$

where $P^{n-1 / 2}(x)$ is a point defined by (3.5). 
Proof Let $X(\cdot ; x)$ be the solution of (3.3). Substituting $(-2 v \nabla D(u)+\nabla p-f)(X(\cdot), \cdot)$ into $f$ and $t^{n}$ into $t$ in (3.2a), using the relation

$$
X\left(t^{n-1} ; x\right)=X_{1}\left(u^{n-1}, \Delta t\right)(x)+O\left(\Delta t^{2}\right),
$$

we have

$$
\begin{aligned}
& -v\left\{\nabla D\left(u^{n}\right)+\nabla D\left(u^{n-1}\right) \circ X_{1}\left(u^{n-1}, \Delta t\right)\right\}(x)+\frac{1}{2}\left\{\nabla p^{n}+\nabla p^{n-1} \circ X_{1}\left(u^{n-1}, \Delta t\right)\right\}(x) \\
& -\frac{1}{2}\left\{f^{n}+f^{n-1} \circ X_{1}\left(u^{n-1}, \Delta t\right)\right\}(x) \\
= & -2 v \nabla D(u)+\nabla p-f\}\left(P^{n-1 / 2}(x)\right)+O\left(\Delta t^{2}\right) .
\end{aligned}
$$

Combining (3.11) with Lemma 3.2, we get the result.

Lemma 3.4 Let $u: \Omega \rightarrow \mathbb{R}^{d}$ be a sufficiently smooth function satisfying $\nabla \cdot u=0$ in $\Omega$ and $X_{1}(u, \Delta t)(\Omega) \subset$ $\Omega$. Then for any $v_{h} \in V_{h}$ it holds that

$$
\begin{aligned}
- & \left(\nabla D(u) \circ X_{1}(u, \Delta t), v_{h}\right) \\
& =\left(D(u) \circ X_{1}(u, \Delta t), D\left(v_{h}\right)\right)+\Delta t\left(D_{i j}(u) u_{k, j}, v_{h i, k}\right)+O\left(\Delta t^{2}\right)\left\|v_{h}\right\| .
\end{aligned}
$$

Proof Since $\nabla \cdot u=0$ in $\Omega$, it holds that

$$
\begin{aligned}
& \left(u_{i, j} \circ X_{1}(u, \Delta t), v_{h i, j}\right)=-\left(\left(u_{i, j} \circ X_{1}(u, \Delta t)\right)_{, j}, v_{h i}\right) \\
= & -\left(u_{i, j k} \circ X_{1}(u, \Delta t)\left(\delta_{k j}-u_{k, j} \Delta t\right), v_{h i}\right) \\
= & -\left(u_{i, j j} \circ X_{1}(u, \Delta t), v_{h i}\right)+\Delta t\left(u_{i, j k} \circ X_{1}(u, \Delta t) u_{k, j}, v_{h i}\right) \\
= & -\left(u_{i, j j} \circ X_{1}(u, \Delta t), v_{h i}\right)+\Delta t\left(u_{i, j k} u_{k, j}, v_{h i}\right)+O\left(\Delta t^{2}\right)\left\|v_{h}\right\| \\
= & -\left(u_{i, j j} \circ X_{1}(u, \Delta t), v_{h i}\right)-\Delta t\left(u_{i, j} u_{k, j}, v_{h i, k}\right)+O\left(\Delta t^{2}\right)\left\|v_{h}\right\|,
\end{aligned}
$$

where $\delta_{k j}$ is Kronecker's delta. Similarly we have

$$
\begin{aligned}
& \left(u_{i, j} \circ X_{1}(u, \Delta t), v_{h j, i}\right)=-\left(\left(u_{i, j} \circ X_{1}(u, \Delta t)\right)_{, i}, v_{h j}\right) \\
= & -\left(u_{i, j i} \circ X_{1}(u, \Delta t), v_{h j}\right)-\Delta t\left(u_{i, j} u_{k, i}, v_{h j, k}\right)+O\left(\Delta t^{2}\right)\left\|v_{h}\right\| \\
= & -\left(u_{j, i j} \circ X_{1}(u, \Delta t), v_{h i}\right)-\Delta t\left(u_{j, i} u_{k, j}, v_{h i, k}\right)+O\left(\Delta t^{2}\right)\left\|v_{h}\right\| .
\end{aligned}
$$

Therefore, it holds that

$$
\begin{aligned}
\left(D(u) \circ X_{1}(u, \Delta t), D\left(v_{h}\right)\right) & =\frac{1}{2}\left\{\left(u_{i, j} \circ X_{1}(u, \Delta t), v_{h i, j}\right)+\left(u_{i, j} \circ X_{1}(u, \Delta t), v_{h j, i}\right)\right\} \\
& =-\left(\nabla D(u) \circ X_{1}(u, \Delta t), v_{h}\right)-\Delta t\left(D_{i j}(u) u_{k, j}, v_{h i, k}\right)+O\left(\Delta t^{2}\right)\left\|v_{h}\right\|,
\end{aligned}
$$

which completes the proof.

Proof of Proposition 3.1 Substituting $u^{n-1}$ into $u$ in Lemma 3.4, we have

$$
\begin{aligned}
& -v\left(\nabla D\left(u^{n-1}\right) \circ X_{1}\left(u^{n-1}, \Delta t\right), v_{h}\right) \\
= & v\left\{\left(D\left(u^{n-1}\right) \circ X_{1}\left(u^{n-1}, \Delta t\right), D\left(v_{h}\right)\right)+\Delta t\left(D_{i j}\left(u^{n-1}\right) u_{k, j}^{n-1}, v_{h i, k}\right)\right\}+O\left(\Delta t^{2}\right)\left\|v_{h}\right\| .
\end{aligned}
$$


Obviously it holds that

$$
-v\left(\nabla D\left(u^{n}\right), v_{h}\right)=v\left(D\left(u^{n}\right), D\left(v_{h}\right)\right) .
$$

Combining (3.13) and (3.14) with Lemma 3.3, we have

$$
\begin{aligned}
& \left\langle\mathscr{A}_{h 2}\left(u^{n}, u^{n}, u^{n-1}, p^{n}, p^{n-1}\right)-\mathscr{F}_{h}^{n}(f, u), v_{h}\right\rangle \\
= & \left(\left(\frac{D u}{D t}-\nabla(2 v D(u))+\nabla p-f\right)^{n-1 / 2} \circ Y_{1}\left(u^{n-1}, \Delta t\right), v_{h}\right)+O\left(\Delta t^{2}\right)\left\|v_{h}\right\| .
\end{aligned}
$$

Here we have used (3.2b) again. Since $(u, p)$ is the solution of (2.1), we get (3.1a). The proof of (3.1b) is similar.

\section{Numerical Examples}

In this section we show numerical results in $d=2$ to observe the numerical convergence rate of the scheme. Since the matrix is symmetric, we use the CG method with ILU(0) preconditioner [2] for solving the system of linear equations. In the scheme we have to compute integrals of composite functions such as

$$
\int_{K} u_{h}^{n-1} \circ X_{2}\left(w_{h}^{k-1}, u_{h}^{n-1}, \Delta t\right) v_{h} d x
$$

on triangular elements $K$. The integrand

$$
u_{h}^{n-1} \circ X_{2}\left(w_{h}^{k-1}, u_{h}^{n-1}, \Delta t\right) v_{h}
$$

is not smooth on $K$. It is known that rough numerical integration causes oscillation even in the case that the stability is theoretically proved for a scheme with exact integration, see [21] and [23]. Hence, much attention should be paid to numerical integration of composite functions. Here we use a numerical integration formula of degree five on each triangle [18]. For a given series $\left\{\psi^{n}\right\}_{n=1}^{N_{T}}$ in a normed space $X$, we define

$$
\|\psi\|_{l^{2}(X)} \equiv\left\{\sum_{n=1}^{N_{T}}\left(t^{n}-t^{n-1}\right)\left\|\psi^{n}\right\|_{X}^{2}\right\}^{\frac{1}{2}} .
$$

Example 4.1 In (2.1) we take $\Omega=(0,1)^{2}, T=1$, and five values of $v$,

$$
v=1,10^{-1}, 10^{-2}, 10^{-3}, 10^{-4} \text {. }
$$

The functions $f$ and $u^{0}$ are given so that the exact solution is

$$
\left\{\begin{array}{l}
u_{1}\left(x_{1}, x_{2}, t\right)=\{1+\sin (\pi t)\} \sin ^{2}\left(\pi x_{1}\right) \sin \left(2 \pi x_{2}\right), \\
u_{2}\left(x_{1}, x_{2}, t\right)=-\{1+\sin (\pi t)\} \sin ^{2}\left(\pi x_{2}\right) \sin \left(2 \pi x_{1}\right), \\
p\left(x_{1}, x_{2}, t\right)=\{1+\sin (\pi t)\} \cos \left(\pi x_{1}\right) \cos \left(\pi x_{2}\right) .
\end{array}\right.
$$

We used FreeFEM [9] for mesh generation. Let $N_{\Omega}$ be the division number of each side of $\Omega$ and $h \equiv 1 / N_{\Omega}$ be the representative length of each mesh. Figure 2(left) shows a sample mesh $\left(N_{\Omega}=8\right)$. We solve the problem by the scheme (2.3). Since the convergence rate of the backward Euler scheme of the P2/P1 Galerkin approximation is $O\left(\Delta t+h^{2}\right)$ for the Navier-Stokes equations, e.g., [24], we choose $\Delta t=h$. Furthermore we set $\Delta t_{0}=h^{2}$ and $\varepsilon_{I}=10^{-5}$. We calculated $E r r$ defined by

$$
E r r \equiv \frac{\left\|\Pi_{h}^{(2)} u-u_{h}\right\|_{l^{2}\left(H^{1}(\Omega)^{2}\right)}+\left\|\Pi_{h}^{(1)} p-p_{h}\right\|_{l^{2}\left(L^{2}(\Omega)\right)}}{\left\|u_{h}\right\|_{l^{2}\left(H^{1}(\Omega)^{2}\right)}+\left\|p_{h}\right\|_{l^{2}\left(L^{2}(\Omega)\right)}} .
$$

Figure 2 (right) shows the graph of $E r r$ versus $\Delta t$ in logarithmic scale for $N_{\Omega}=8,16,32$ and 64, and the values of Err and the slopes are given in Table 1. We can observe a second order convergence in $\Delta t$. 


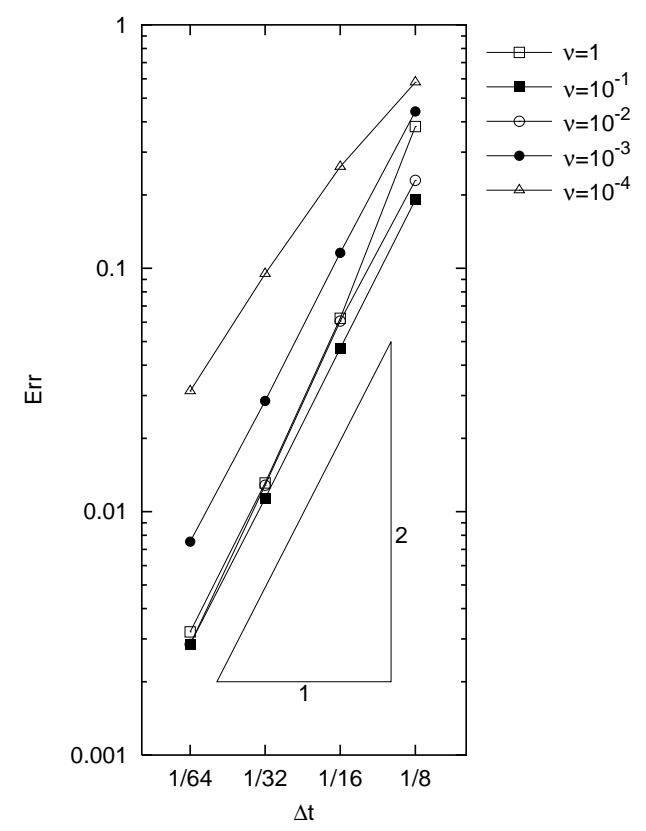

Fig. 2: A sample mesh $\left(N_{\Omega}=8\right)$ and the graph of $\operatorname{Err}$ versus $\Delta t$ in logarithmic scale

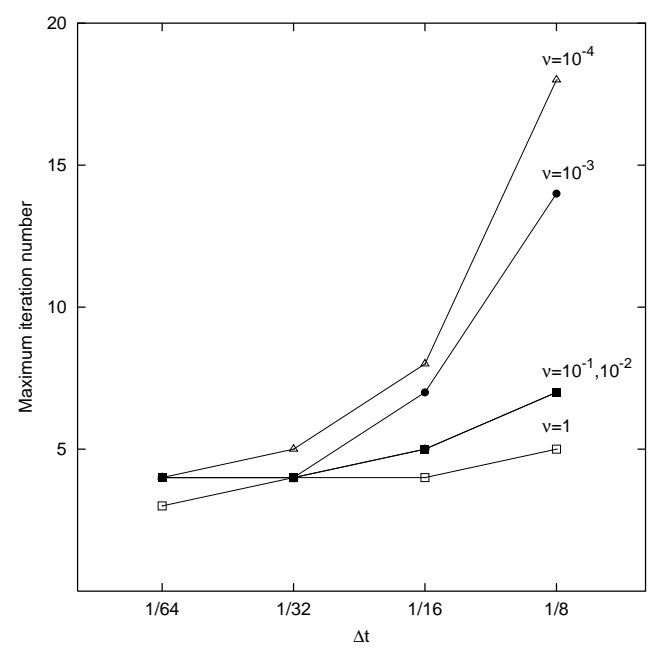

Fig. 3: The graph of maximum iteration number versus $\Delta t$ for each $v$

Figure 3 exhibits the graph of maximum internal iteration number versus $\Delta t$. It decreases as $\Delta t$ becomes small and was equal to 3 or 4 for $\Delta t=1 / 64$.

Now we examine the importance of the additional correction term

$$
v \Delta t\left(D_{i j}\left(u^{n-1}\right) u_{k, j}^{n-1}, v_{h i, k}\right)
$$

in the definition of $\mathscr{D}_{h 2}$. We compare results obtained by schemes with and without this term as well as the first order scheme. Dropping the term from the scheme (2.3), we get

$$
\left\{\begin{aligned}
\hat{\mathscr{A}}_{h}^{n}\left(u_{h}, p_{h}\right) & =\mathscr{F}_{h}^{n}\left(f_{h}, u_{h}\right) & & \text { in } V_{h}^{\prime}, \\
\mathscr{B}_{h}^{n} u_{h} & =0 & & \text { in } Q_{h}^{\prime}, \\
u_{h}^{0} & =\Pi_{h}^{(2)} u^{0}, & &
\end{aligned}\right.
$$


where

$$
\begin{aligned}
\hat{\mathscr{A}}_{h}^{n}(u, p) & \equiv \begin{cases}\hat{\mathscr{A}}_{h 2}\left(u^{n}, u^{n}, u^{n-1}, p^{n}, p^{n-1}\right) & (n \geq 2), \\
\mathscr{A}_{h 1}\left(u^{1}, u^{0}, p^{1}\right) & (n=1),\end{cases} \\
\hat{\mathscr{A}}_{h 2}(u, \zeta, w, p, q) & \equiv \mathscr{M}_{h 2}(u, \zeta, w ; \Delta t)+\hat{\mathscr{D}}_{h 2}(u, w)+\mathscr{C}_{h 2}(w, p, q), \\
\left\langle\hat{\mathscr{D}}_{h 2}(u, w), v_{h}\right\rangle & \equiv v\left(D(u)+D(w) \circ X_{1}(w, \Delta t), D\left(v_{h}\right)\right) .
\end{aligned}
$$

The first order scheme is

$$
\left\{\begin{aligned}
\mathscr{A}_{h 1}^{n}\left(u_{h}, p_{h}\right) & =\mathscr{F}_{h 1}^{n} f_{h} & & \text { in } V_{h}^{\prime}, \\
\mathscr{B}_{h}^{n} u_{h} & =0 & & \text { in } Q_{h}^{\prime}, \\
u_{h}^{0} & =\Pi_{h}^{(2)} u^{0}, & &
\end{aligned}\right.
$$

where

$$
\begin{aligned}
\mathscr{A}_{h 1}^{n}(u, p) & \equiv \begin{cases}\mathscr{M}_{h 1}\left(u^{n}, u^{n-1} ; \Delta t\right)+\mathscr{D}_{h 1} u^{n}+\mathscr{C}_{h 1} p^{n} & (n \geq 2), \\
\mathscr{M}_{h 1}\left(u^{1}, u^{0} ; \Delta t_{0}\right)+\mathscr{D}_{h 1} u^{1}+\mathscr{C}_{h 1} p^{1} & (n=1) .\end{cases} \\
\mathscr{F}_{h 1}^{n} f & \equiv \mathscr{F}_{h 1} f^{n} .
\end{aligned}
$$

In the first order scheme we do not need to use a first step with a small time increment $\Delta t_{0}$. For the comparison with other schemes, however, we use the first step with $\Delta t_{0}$. We solve Example 4.1 under the same condition. The results obtained from the three schemes are shown in Figure 4 and Table 1. These results exhibit the necessity of the additional correction term for second order in $\Delta t$. In the case of $v=1$ the values of $E r r$ of the scheme (4.2) are worse than those of the scheme (4.3). In the case of $v=10^{-1}$ the results of (4.2) is better than those of (4.3), but the slope of (4.2) is worse than that of the present scheme (2.3). In the cases $v=10^{-2}, 10^{-3}$ and $10^{-4}$ there is no clear difference between the results by (4.2) and (2.3). These results are explained from the fact that the additional correction term (4.1) contains $v$ and is proportional to it.

\section{Conclusions}

We have presented a new characteristic-curve finite element scheme of single step and second order in $\Delta t$ for the Navier-Stokes equations. We have shown an additional correction term for the scheme to be of second order in the time increment. Our approximation is based on the Crank-Nicolson method on the trajectory, which is the reason why the correction term is required. Since the scheme is nonlinear, we presented an internal iteration procedure. In each internal iteration the matrix is symmetric and identical. In our numerical examples the internal iteration has converged as few as three or four steps when the time increment is small. We have also given numerical results which confirm the second order accuracy in $\Delta t$ and the importance of the additional correction term. To make a stable scheme in a weak form of the pressure is an open problem.

Acknowledgements This work was supported by the Japan Society for the Promotion of Science under Grant-in-Aid for Scientific Research (S), No. 16104001 and the Ministry of Education, Culture, Sports, Science and Technology of Japan under Kyushu University 21st Century COE Program, Development of Dynamic Mathematics with High Functionality.

\section{References}

[1] Baba, K., and Tabata, M. (1981). On a conservative upwind finite element scheme for convective diffusion equations. RAIRO Anal. Numér. 15, 3-25. 

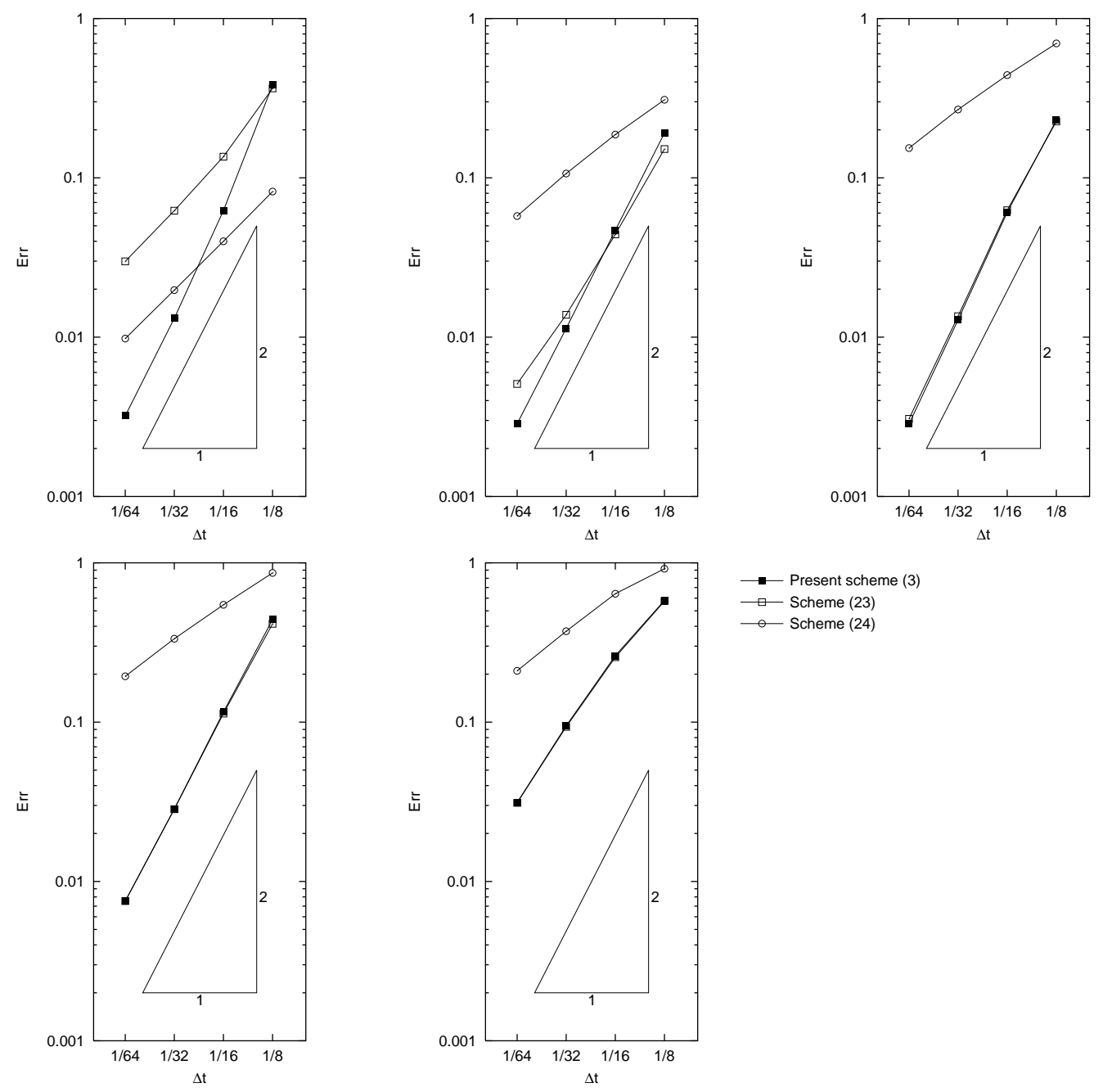

Fig. 4: Comparison of convergence order: $v=1$ (top left), $10^{-1}$ (top center), $10^{-2}$ (top right), $10^{-3}$ (bottom left) and $10^{-4}$ (bottom right)

[2] Barrett, R., Berry, M., Chan, T. F., Demmel, J., Donato, J., Dongarra, J., Eijkhout, V., Pozo, R., Romine, C., and Van der Vorst, H. (1994). Templates for the Solution of Linear Systems: Building Blocks for Iterative Methods, SIAM.

[3] Bristeau, M. O., Glowinski, R., Mantel,B., Periaux, J., Perrier, P. and Pironneau, O. (1980). A finite element approximation of Navier-Stokes equations for incompressible viscous fluids. Iterative methods of solution. In R. Rautmann, editor, Approximation Methods for Navier-Stokes Problems, 771, Lecture Notes in Mathematics, 78-128. Springer, Berlin.

[4] Brooks, A. N., and Hughes, T. J. R. (1982). Streamline upwind/Petrov-Galerkin formulations for convection dominated flows with particular emphasis on the incompressible Navier-Stokes equations. Comput. Methods. Appl. Mech. Engrg. 32, 199-259.

[5] Boukir, K., Maday, Y., Metivet, B., and Razafindrakoto, E. (1997). A high-order characteristic/finite element method for the incompressible Navier-Stokes equations. Int. J. Numer. Methods Fluids 25, $1421-1454$.

[6] Brezzi, F. and Fortin, M. (1982). Mixed and Hybrid Finite Element Methods. Springer, New York, 1991. 
Table 1: Values of Err and slopes of the graphs in Figures 2 and 4

\begin{tabular}{|c|c|c|c|c|c|c|c|}
\hline & \multirow[b]{2}{*}{$N_{\Omega}$} & \multicolumn{2}{|c|}{ Present scheme (2.3) } & \multicolumn{2}{|c|}{ Scheme (4.2) } & \multicolumn{2}{|c|}{ Scheme (4.3) } \\
\hline & & Err & slope & Err & slope & Err & slope \\
\hline \multirow[t]{4}{*}{$v=1:$} & $\overline{8}$ & $3.82 \times 10^{-1}$ & - & $3.64 \times 10^{-1}$ & - & $8.20 \times 10^{-2}$ & - \\
\hline & 16 & $6.23 \times 10^{-2}$ & 2.62 & $1.36 \times 10^{-1}$ & 1.42 & $4.00 \times 10^{-2}$ & 1.04 \\
\hline & 32 & $1.31 \times 10^{-2}$ & 2.25 & $6.22 \times 10^{-2}$ & 1.13 & $1.97 \times 10^{-2}$ & 1.02 \\
\hline & 64 & $3.21 \times 10^{-3}$ & 2.03 & $2.99 \times 10^{-2}$ & 1.06 & $9.80 \times 10^{-3}$ & 1.01 \\
\hline \multirow[t]{4}{*}{$v=10^{-1}:$} & 8 & $1.91 \times 10^{-1}$ & - & $1.51 \times 10^{-1}$ & - & $3.09 \times 10^{-1}$ & - \\
\hline & 16 & $4.68 \times 10^{-2}$ & 2.03 & $4.41 \times 10^{-2}$ & 1.78 & $1.87 \times 10^{-1}$ & 0.73 \\
\hline & 32 & $1.13 \times 10^{-2}$ & 2.05 & $1.38 \times 10^{-2}$ & 1.68 & $1.06 \times 10^{-1}$ & 0.81 \\
\hline & 64 & $2.86 \times 10^{-3}$ & 1.99 & $5.08 \times 10^{-3}$ & 1.44 & $5.76 \times 10^{-2}$ & 0.89 \\
\hline \multirow[t]{4}{*}{$v=10^{-2}:$} & 8 & $2.30 \times 10^{-1}$ & - & $2.26 \times 10^{-1}$ & - & $6.98 \times 10^{-1}$ & - \\
\hline & 16 & $6.07 \times 10^{-2}$ & 1.92 & $6.26 \times 10^{-2}$ & 1.85 & $4.41 \times 10^{-1}$ & 0.66 \\
\hline & 32 & $1.28 \times 10^{-2}$ & 2.24 & $1.35 \times 10^{-2}$ & 2.21 & $2.68 \times 10^{-1}$ & 0.72 \\
\hline & 64 & $2.85 \times 10^{-3}$ & 2.17 & $3.07 \times 10^{-3}$ & 2.14 & $1.54 \times 10^{-1}$ & 0.80 \\
\hline \multirow[t]{4}{*}{$v=10^{-3}:$} & 8 & $4.41 \times 10^{-1}$ & - & $4.14 \times 10^{-1}$ & - & $8.65 \times 10^{-1}$ & - \\
\hline & 16 & $1.16 \times 10^{-1}$ & 1.93 & $1.13 \times 10^{-1}$ & 1.87 & $5.45 \times 10^{-1}$ & 0.67 \\
\hline & 32 & $2.85 \times 10^{-2}$ & 2.02 & $2.84 \times 10^{-2}$ & 2.00 & $3.34 \times 10^{-1}$ & 0.71 \\
\hline & 64 & $7.53 \times 10^{-3}$ & 1.92 & $7.53 \times 10^{-3}$ & 1.92 & $1.94 \times 10^{-1}$ & 0.78 \\
\hline \multirow[t]{4}{*}{$v=10^{-4}:$} & 8 & $5.81 \times 10^{-1}$ & - & $5.75 \times 10^{-1}$ & - & $9.18 \times 10^{-1}$ & - \\
\hline & 16 & $2.61 \times 10^{-1}$ & 1.15 & $2.56 \times 10^{-1}$ & 1.17 & $6.39 \times 10^{-1}$ & 0.52 \\
\hline & 32 & $9.48 \times 10^{-2}$ & 1.46 & $9.35 \times 10^{-2}$ & 1.45 & $3.72 \times 10^{-1}$ & 0.78 \\
\hline & 64 & $3.13 \times 10^{-2}$ & 1.60 & $3.11 \times 10^{-2}$ & 1.59 & $2.10 \times 10^{-1}$ & 0.83 \\
\hline
\end{tabular}

[7] Douglas Jr., J., and Russell, T. F. (1982). Numerical methods for convection-dominated diffusion problems based on combining the method of characteristic with finite element or finite difference procedures. SIAM J. Numer. Math. 38, 309-332.

[8] Franca, L. P. and Frey, S. L. (1992). Stabilized finite element methods: II. The incompressible Navier-Stokes equations. Computer Methods in Applied Mechanics and Engineering, 99, 209-233.

[9] FreeFem, http://www.freefem.org/.

[10] Girault, V., and Raviart, P. A. (1986). Finite Element Methods for Navier-Stokes Equations, Springer Verlag, Berlin-Heidelberg.

[11] Hughes, T. J. R., Franca, L. P., and Hulbert, G. M. (1989). A new finite element formulation for computational fluid dynamics: VIII. The Galerkin/least-squares method for advective-diffusive equations. Comput. Methods. Appl. Mech. Engrg. 73, 173-189.

[12] Hansbo, P., and Johnson, C. (1990). Adaptive streamline diffusion methods for compressible flow using conservation variables. Comput. Methods. Appl. Mech. Engrg. 87, 267-280.

[13] Johnson, C. (1987). Numerical Solution of Partial Differential Equations by the Finite Element Method. Cambridge Univ. Press, Cambridge.

[14] Pironneau, O. (1982). On the transport-diffusion algorithm and its application to the Navier-Stokes equations. Numer. Math. 38, 309-332.

[15] Pironneau, O. (1989). Finite Element Methods for Fluids, John Wiley \& Sons, Chichester. 
[16] Pironneau, O., Liou, J. and Tezduyar, T. (1992). Characteristic-Galerkin and Galerkin/least-squares space-time formulations for the advection-diffusion equation with time-dependent domains. Computer Methods in Applied Mechanics and Engineering, 100, 117-141.

[17] Rui, H., and Tabata, M. (2002). A second order characteristic finite element scheme for convectiondiffusion problems. Numer. Math. 92, 161-177.

[18] Stroud, A. H. (1971). Approximate calculation of multiple integrals, Prentice-Hall.

[19] Süli, E. (1988). Convergence and nonlinear stability of the Lagrange-Galerkin method for the Navier-Stokes equations. Numer. Math. 53, 459-483.

[20] Tabata, M. (1977). A finite element approximation corresponding to the upwind differencing. Mem. Numer. Math. 4, 47-63.

[21] Tabata, M. (2007). Discrepancy between theory and real computation on the stability of some finite element schemes. J. Comput. App. Math. 199, 424-431.

[22] Tabata, M. and Fujima, S. (1991). Finite-element analysis of high Reynolds number flows past a circular cylinder. Journal of Computational and Applied Mathematics, 38, 411-424.

[23] Tabata, M., and Fujima, S. (2006). Robustness of a characteristic finite element scheme of second order in time increment. In Groth, C., and Zingg, D.W. (eds.), Proc. Third International Conference on Computational Fluid Dynamics, Springer Verlag, Berlin-Heidelberg, pp. 177-182.

[24] Tabata, M and Tagami, D. (2000). Error estimates for finite element approximations of drag and lift in nonstationary Navier-Stokes flows. Japan Journal of Industrial and Applied Mathematics, Vol. 17, pp. 371-389.

[25] Thomasset, F. (1981). Implementation of Finite Element Methods for Navier-Stokes Equations. Springer, New York. 


\section{List of MHF Preprint Series, Kyushu University \\ 21st Century COE Program \\ Development of Dynamic Mathematics with High Functionality}

MHF2005-1 Hideki KOSAKI

Matrix trace inequalities related to uncertainty principle

MHF2005-2 Masahisa TABATA

Discrepancy between theory and real computation on the stability of some finite element schemes

MHF2005-3 Yuko ARAKI \& Sadanori KONISHI

Functional regression modeling via regularized basis expansions and model selection

MHF2005-4 Yuko ARAKI \& Sadanori KONISHI

Functional discriminant analysis via regularized basis expansions

MHF2005-5 Kenji KAJIWARA, Tetsu MASUDA, Masatoshi NOUMI, Yasuhiro OHTA \& Yasuhiko YAMADA

Point configurations, Cremona transformations and the elliptic difference Painlevé equations

MHF2005-6 Kenji KAJIWARA, Tetsu MASUDA, Masatoshi NOUMI, Yasuhiro OHTA \& Yasuhiko YAMADA

Construction of hypergeometric solutions to the $q$ - Painlevé equations

MHF2005-7 Hiroki MASUDA

Simple estimators for non-linear Markovian trend from sampled data:

I. ergodic cases

MHF2005-8 Hiroki MASUDA \& Nakahiro YOSHIDA

Edgeworth expansion for a class of Ornstein-Uhlenbeck-based models

MHF2005-9 Masayuki UCHIDA

Approximate martingale estimating functions under small perturbations of dynamical systems

MHF2005-10 Ryo MATSUZAKI \& Masayuki UCHIDA

One-step estimators for diffusion processes with small dispersion parameters from discrete observations

MHF2005-11 Junichi MATSUKUBO, Ryo MATSUZAKI \& Masayuki UCHIDA

Estimation for a discretely observed small diffusion process with a linear drift

MHF2005-12 Masayuki UCHIDA \& Nakahiro YOSHIDA

AIC for ergodic diffusion processes from discrete observations 
MHF2005-13 Hiromichi GOTO \& Kenji KAJIWARA

Generating function related to the Okamoto polynomials for the Painlevé IV equation

MHF2005-14 Masato KIMURA \& Shin-ichi NAGATA

Precise asymptotic behaviour of the first eigenvalue of Sturm-Liouville problems with large drift

MHF2005-15 Daisuke TAGAMI \& Masahisa TABATA

Numerical computations of a melting glass convection in the furnace

MHF2005-16 Raimundas VIDŪNAS

Normalized Leonard pairs and Askey-Wilson relations

MHF2005-17 Raimundas VIDŪNAS

Askey-Wilson relations and Leonard pairs

MHF2005-18 Kenji KAJIWARA \& Atsushi MUKAIHIRA

Soliton solutions for the non-autonomous discrete-time Toda lattice equation

MHF2005-19 Yuu HARIYA

Construction of Gibbs measures for 1-dimensional continuum fields

MHF2005-20 Yuu HARIYA

Integration by parts formulae for the Wiener measure restricted to subsets in $\mathbb{R}^{d}$

MHF2005-21 Yuu HARIYA

A time-change approach to Kotani's extension of Yor's formula

MHF2005-22 Tadahisa FUNAKI, Yuu HARIYA \& Mark YOR

Wiener integrals for centered powers of Bessel processes, I

MHF2005-23 Masahisa TABATA \& Satoshi KAIZU

Finite element schemes for two-fluids flow problems

MHF2005-24 Ken-ichi MARUNO \& Yasuhiro OHTA

Determinant form of dark soliton solutions of the discrete nonlinear Schrödinger equation

MHF2005-25 Alexander V. KITAEV \& Raimundas VIDŪNAS

Quadratic transformations of the sixth Painlevé equation

MHF2005-26 Toru FUJII \& Sadanori KONISHI

Nonlinear regression modeling via regularized wavelets and smoothing parameter selection

MHF2005-27 Shuichi INOKUCHI, Kazumasa HONDA, Hyen Yeal LEE, Tatsuro SATO, Yoshihiro MIZOGUCHI \& Yasuo KAWAHARA

On reversible cellular automata with finite cell array 
MHF2005-28 Toru KOMATSU

Cyclic cubic field with explicit Artin symbols

MHF2005-29 Mitsuhiro T. NAKAO, Kouji HASHIMOTO \& Kaori NAGATOU

A computational approach to constructive a priori and a posteriori error estimates for finite element approximations of bi-harmonic problems

MHF2005-30 Kaori NAGATOU, Kouji HASHIMOTO \& Mitsuhiro T. NAKAO

Numerical verification of stationary solutions for Navier-Stokes problems

MHF2005-31 Hidefumi KAWASAKI

A duality theorem for a three-phase partition problem

MHF2005-32 Hidefumi KAWASAKI

A duality theorem based on triangles separating three convex sets

MHF2005-33 Takeaki FUCHIKAMI \& Hidefumi KAWASAKI

An explicit formula of the Shapley value for a cooperative game induced from the conjugate point

MHF2005-34 Hideki MURAKAWA

A regularization of a reaction-diffusion system approximation to the two-phase

Stefan problem

MHF2006-1 Masahisa TABATA

Numerical simulation of Rayleigh-Taylor problems by an energy-stable finite element scheme

MHF2006-2 Ken-ichi MARUNO \& G R W QUISPEL

Construction of integrals of higher-order mappings

MHF2006-3 Setsuo TANIGUCHI

On the Jacobi field approach to stochastic oscillatory integrals with quadratic phase function

MHF2006-4 Kouji HASHIMOTO, Kaori NAGATOU \& Mitsuhiro T. NAKAO

A computational approach to constructive a priori error estimate for finite element approximations of bi-harmonic problems in nonconvex polygonal domains

MHF2006-5 Hidefumi KAWASAKI

A duality theory based on triangular cylinders separating three convex sets in $R^{n}$

MHF2006-6 Raimundas VIDŪNAS

Uniform convergence of hypergeometric series

MHF2006-7 Yuji KODAMA \& Ken-ichi MARUNO

N-Soliton solutions to the DKP equation and Weyl group actions 
MHF2006-8 Toru KOMATSU

Potentially generic polynomial

MHF2006-9 Toru KOMATSU

Generic sextic polynomial related to the subfield problem of a cubic polynomial

MHF2006-10 Shu TEZUKA \& Anargyros PAPAGEORGIOU

Exact cubature for a class of functions of maximum effective dimension

MHF2006-11 Shu TEZUKA

On high-discrepancy sequences

MHF2006-12 Raimundas VIDŪNAS

Detecting persistent regimes in the North Atlantic Oscillation time series

MHF2006-13 Toru KOMATSU

Tamely Eisenstein field with prime power discriminant

MHF2006-14 Nalini JOSHI, Kenji KAJIWARA \& Marta MAZZOCCO

Generating function associated with the Hankel determinant formula for the solutions of the Painlevé IV equation

MHF2006-15 Raimundas VIDŪNAS

Darboux evaluations of algebraic Gauss hypergeometric functions

MHF2006-16 Masato KIMURA \& Isao WAKANO

New mathematical approach to the energy release rate in crack extension

MHF2006-17 Toru KOMATSU

Arithmetic of the splitting field of Alexander polynomial

MHF2006-18 Hiroki MASUDA

Likelihood estimation of stable Lévy processes from discrete data

MHF2006-19 Hiroshi KAWABI \& Michael RÖCKNER

Essential self-adjointness of Dirichlet operators on a path space with Gibbs measures via an SPDE approach

MHF2006-20 Masahisa TABATA

Energy stable finite element schemes and their applications to two-fluid flow problems

MHF2006-21 Yuzuru INAHAMA \& Hiroshi KAWABI

Asymptotic expansions for the Laplace approximations for Itô functionals of Brownian rough paths

MHF2006-22 Yoshiyuki KAGEI

Resolvent estimates for the linearized compressible Navier-Stokes equation in an infinite layer 
MHF2006-23 Yoshiyuki KAGEI

Asymptotic behavior of the semigroup associated with the linearized compressible Navier-Stokes equation in an infinite layer

MHF2006-24 Akihiro MIKODA, Shuichi INOKUCHI, Yoshihiro MIZOGUCHI \& Mitsuhiko FUJIO

The number of orbits of box-ball systems

MHF2006-25 Toru FUJII \& Sadanori KONISHI

Multi-class logistic discrimination via wavelet-based functionalization and model selection criteria

MHF2006-26 Taro HAMAMOTO, Kenji KAJIWARA \& Nicholas S. WITTE

Hypergeometric solutions to the $q$-Painlevé equation of type $\left(A_{1}+A_{1}^{\prime}\right)^{(1)}$

MHF2006-27 Hiroshi KAWABI \& Tomohiro MIYOKAWA

The Littlewood-Paley-Stein inequality for diffusion processes on general metric spaces

MHF2006-28 Hiroki MASUDA

Notes on estimating inverse-Gaussian and gamma subordinators under highfrequency sampling

MHF2006-29 Setsuo TANIGUCHI

The heat semigroup and kernel associated with certain non-commutative harmonic oscillators

MHF2006-30 Setsuo TANIGUCHI

Stochastic analysis and the KdV equation

MHF2006-31 Masato KIMURA, Hideki KOMURA, Masayasu MIMURA, Hidenori MIYOSHI, Takeshi TAKAISHI \& Daishin UEYAMA

Quantitative study of adaptive mesh FEM with localization index of pattern

MHF2007-1 Taro HAMAMOTO \& Kenji KAJIWARA

Hypergeometric solutions to the $q$-Painlevé equation of type $A_{4}^{(1)}$

MHF2007-2 Kouji HASHIMOTO, Kenta KOBAYASHI \& Mitsuhiro T. NAKAO

Verified numerical computation of solutions for the stationary Navier-Stokes equation in nonconvex polygonal domains

MHF2007-3 Kenji KAJIWARA, Marta MAZZOCCO \& Yasuhiro OHTA

A remark on the Hankel determinant formula for solutions of the Toda equation

MHF2007-4 Jun-ichi SATO \& Hidefumi KAWASAKI

Discrete fixed point theorems and their application to Nash equilibrium

MHF2007-5 Mitsuhiro T. NAKAO \& Kouji HASHIMOTO

Constructive error estimates of finite element approximations for non-coercive elliptic problems and its applications 
MHF2007-6 Kouji HASHIMOTO

A preconditioned method for saddle point problems

MHF2007-7 Christopher MALON, Seiichi UCHIDA \& Masakazu SUZUKI

Mathematical symbol recognition with support vector machines

MHF2007-8 Kenta KOBAYASHI

On the global uniqueness of Stokes' wave of extreme form

MHF2007-9 Kenta KOBAYASHI

A constructive a priori error estimation for finite element discretizations in a non-convex domain using singular functions

MHF2007-10 Myoungnyoun KIM, Mitsuhiro T. NAKAO, Yoshitaka WATANABE \& Takaaki NISHIDA

A numerical verification method of bifurcating solutions for 3-dimensional Rayleigh-Bénard problems

MHF2007-11 Yoshiyuki KAGEI

Large time behavior of solutions to the compressible Navier-Stokes equation in an infinite layer

MHF2007-12 Takashi YANAGAWA, Satoshi AOKI and Tetsuji OHYAMA

Human finger vein images are diverse and its patterns are useful for personal identification

MHF2007-13 Masahisa TABATA

Finite element schemes based on energy-stable approximation for two-fluid flow problems with surface tension

MHF2007-14 Mitsuhiro T. NAKAO \& Takehiko KINOSHITA

Some remarks on the behaviour of the finite element solution in nonsmooth domains

MHF2007-15 Yoshiyuki KAGEI \& Takumi NUKUMIZU

Asymptotic behavior of solutions to the compressible Navier-Stokes equation in a cylindrical domain

MHF2007-16 Shuichi INOKUCHI, Yoshihiro MIZOGUCHI, Hyen Yeal LEE \& Yasuo KAWAHARA

Periodic behaviors of quantum cellular automata

MHF2007-17 Makoto HIROTA\& Yasuhide FUKUMOTO

Energy of hydrodynamic and magnetohydrodynamic waves with point and continuous spectra

MHF2007-18 Mitsunori KAYANO\& Sadanori KONISHI

Functional principal component analysis via regularized Gaussian basis expansions and its application to unbalanced data 
MHF2007-19 Mitsunori KAYANO, Koji DOZONO \& Sadanori KONISHI

Functional cluster analysis via orthonormalized Gaussian basis expansions and its application

MHF2008-1 Jun-ichi SATO

An application of a discrete fixed point theorem to the Cournot model

MHF2008-2 Kei HIROSE, Shuichi KAWANO, Sadanori KONISHI \& Masanori ICHIKAWA Bayesian factor analysis and model selection

MHF2008-3 Yoshitaka WATANABE, Michael PLUM \& Mitsuhiro T. NAKAO

A computer-assisted instability proof for the Orr-Sommerfeld problem with Poiseuille flow

MHF2008-4 Hirofumi NOTSU \& Masahisa TABATA

A characteristic-curve finite element scheme of single step and second order in time increment for the Navier-Stokes equations 